\title{
Identificación de agentes infecciosos asociados con Diarrea Neonatal Bovina en la Sabana de Bogotá
}

\section{Identification of infectious agents associated with Bovine Neonatal Diarrhea in the Sabana de Bogotá}

\author{
Dolly Pardo M, ${ }^{1}$ M.Sc, Olimpo Oliver ${ }_{,}{ }^{1 *}$ Ph.D.
}

\begin{abstract}
${ }^{1}$ Universidad Nacional de Colombia, Facultad de Medicina Veterinaria y de Zootecnia Departamento de Ciencias para la Salud Animal, Grupo de Investigación en Enfermedades de Grandes Animales, Bogotá, Colombia. *Correspondencia: ojolivere@unal.edu.co
\end{abstract}

Recibido: Mayo de 2011; Aceptado: Febrero de 2012.

\section{RESUMEN}

Objetivo. Determinar los agentes infecciosos asociados causalmente a la presentación de Diarrea Neonatal Bovina (DNB) en terneros menores de 5 semanas de vida, procedentes de fincas lecheras y de producción mixta de la Sabana de Bogotá. Materiales y métodos. Se seleccionaron por conveniencia 21 fincas, se realizó seguimiento de 620 terneros desde el nacimiento hasta las 5 semanas de edad, se tomaron muestras de materia fecal de los animales que presentaron cuadro clínico de diarrea y de terneros clínicamente sanos como controles pareados. Se realizaron pruebas de ELISA para diagnóstico de E.coli F5, Rotavirus, Coronavirus, Cryptosporidium sp., y Salmonella sp., prueba de Ritchie para diagnóstico de Giardia sp., y tinción de Ziehl Neelsen modificada para Cryptosporidium sp. Se evaluó la asociación epidemiológica entre los agentes y la presentación de diarrea usando prueba de $\chi^{2}$, seguido de un modelo de regresión logística $(p<0.05)$. Resultados. Se encontró en la prueba de ELISA que de la totalidad de las muestras, $51(38.3 \%), 26(19.7 \%), 10(7.5 \%)$ y $1(0.75 \%)$ fueron positivas a Cryptosporidium sp., rotavirus, E coli $\mathrm{F} 5$ y coronavirus, respectivamente. Los animales positivos a Rotavirus por la prueba de ELISA y a Cryptosporidium sp., por la técnica de Ziehl Neelsen modificada tuvieron 2.6 y 7.0 veces mayor probabilidad que los demás animales de presentar DNB, respectivamente. Conclusiones. Los resultados presentados son los primeros que muestran el papel y la importancia del Cryptosporidium sp., y del Rotavirus en la DNB en las explotaciones ganaderas de la Sabana de Bogotá y en Colombia.

Palabras clave: Diarrea, rotavirus, Cryptosporidium, prueba de ELISA (Fuente: DeCS). 


\begin{abstract}
Objective. To determine the infectious agents causally associated to Bovine Neonatal Diarrhea (BND) in calves younger than five weeks of age from mixed production and dairy herds of Sabana de Bogota. Materials and methods. Twenty one herds were conveniently selected, and 620 calves were followed from birth up to 5 weeks of age, fecal samples were collected from animals with clinical signs of diarrhea and from calves without diarrhea which were taken as matched controls. ELISA tests were performed to diagnose E. coli F5, Rotavirus, Coronavirus, Salmonella sp, and Cryptosporidium $\mathrm{sp}$, Ritchie test to diagnose Giardia sp., and a modified Ziehl Neelsen staining for Cryptosporidium $\mathrm{sp}$. The epidemiological association between agents and the appearance of diarrhea was evaluated using $\chi^{2}$ test, followed by a logistic regression model $(p<0.05)$. Results. The ELISA test showed that $51(38.3 \%), 26(19.7 \%), 10(7.5 \%)$ and $1(0.75 \%)$ samples were positive for Cryptosporidium sp., rotavirus, E coli $\mathrm{F} 5$ and coronavirus, respectively. Animals that were positive for Rotavirus through ELISA and Cryptosporidium sp., through a modified Ziehl Neelsen technique had 2.6 and 7.0 times more probability than other animals to present BND, respectively. Conclusions. The results presented are the first to show the importance of Rotavirus and Cryptosporidium sp., in BND in the Sabana de Bogotá herds and in Colombia.
\end{abstract}

Key words: Diarrhea, rotavirus, Cryptosporidium, ELISA test (Source: DeCS).

\title{
INTRODUCCIÓN
}

Diarrea Neonatal Bovina (DNB) es una enfermedad compleja y multifactorial que ocurre como consecuencia de la interacción de factores relacionados con la vaca, el ternero, el estado inmune, las prácticas de manejo, los factores ambientales y la infección con enteropatógenos (1-2).

Se ha investigado acerca de la fisiopatología de la diarrea infecciosa durante muchas décadas, esto ha llevado a un mejor entendimiento de la entidad; sin embargo, a pesar del mejoramiento de las prácticas de manejo, la prevención y las estrategias de tratamiento, esta enfermedad continua siendo muy común y altamente costosa (3).

Se considera que existen cinco agentes enteropatógenos principales y más comunes en la DNB: Echerichia coli enterotoxigenica, rotavirus, coronavirus, Cryptosporidium sp., y Salmonella spp. La prevalencia relativa de estos agentes varía bastante entre los diferentes estudios realizados, posiblemente por diferencias en la ubicación, el clima, las técnicas de diagnóstico y otros factores (2-4). Hay otros enteropatógenos menos comunes como $E$. coli verotoxigénica (VTEC), E.coli necrotoxigénica (NTEC), Giardia duodenalis, Torovirus, Calicivirus y Norovirus (4).

Para establecer medidas eficientes de prevención y control es indispensable determinar la causa específica de esta enfermedad. En los casos de diarrea el color, la consistencia y otras características físicas pueden verse similares por eso la identificación del agente es necesaria para hacer el diagnóstico (1).
En Colombia los estudios realizados reportan incidencias de DNB del $26.1 \%$ y el $37.5 \%$ para los departamentos de Cundinamarca y Antioquia respectivamente $(5,6)$; indicando que la enfermedad es de gran importancia en el país.

El objetivo del estudio fue determinar los agentes infecciosos asociados de manera casual con la Diarrea Neonatal Bovina en terneros menores de 5 semanas de vida y procedentes de fincas lecheras y de producción mixta de la Sabana de Bogotá.

\section{MATERIALES Y MÉTODOS}

Selección de las fincas. Se seleccionaron por conveniencia 21 fincas ubicadas en 15 municipios de la Sabana de Bogotá (Tabla 1), entre febrero de 2009 y enero de 2010. Quince fincas lecheras, dos de doble propósito y tres de producción mixta (con animales de razas lecheras y razas de carne).

Tabla 1. Ubicación de las 21 fincas ganaderas en la Sabana de Bogota.

\begin{tabular}{lccc}
\hline \multicolumn{1}{c}{ Municipio } & No. De fincas & Municipio & No. De fincas \\
\hline Soacha & 1 & Choconta & 2 \\
Sibate & 1 & Villapinzón & 2 \\
Funza & 3 & Zipaquira & 2 \\
Tenjo & 1 & El Rosal & 1 \\
Cota & 1 & Guasca & 1 \\
Chia & 2 & Nemocón & 1 \\
Sopo & 1 & Suesca & 1 \\
Gachancipa & 1 & & \\
\hline
\end{tabular}


Inicialmente se realizó una encuesta en cada finca con el fin de obtener datos relacionados con el estado de salud, productivo, reproductivo y manejo general a nivel de finca y a nivel de ternero. De manera adicional, de cada parto se tomaron los datos relacionados con la madre, la historia del parto y el estado del ternero recién nacido mediante la realización de una encuesta individual al personal encargado de la atención del parto. Los terneros fueron pesados con cinta bovinométrica y cada uno fue identificado de acuerdo al sistema propio de cada finca.

Se realizaron visitas para el seguimiento a cada ternero durante sus primeros 35 días de vida; cuando algún ternero presentó el cuadro clínico de DNB (caracterizado por la evacuación de heces fluidas, frecuentes, abundantes, anorexia, deshidratación) fue incluido en el estudio y se definió como caso e inmediatamente se escogieron uno o dos controles pareados que correspondían a terneros sanos de la misma finca y que tuvieran una edad similar (diferencia menor a 7 días). De los casos y los controles se tomaron muestras de materia fecal directamente del recto, previo a la aplicación de tratamiento de antibióticos en la finca, estas muestras fueron procesadas para las pruebas parasitológicas y luego se mantuvieron a $-70^{\circ} \mathrm{C}$ hasta el momento de la realización de las pruebas de diagnóstico.

Pruebas de laboratorio. Para diagnosticar la presencia o ausencia de los enteropatógenos E.coli F5, Rotavirus, Coronavirus, Cryptosporidium parvum en las muestras de materia fecal de los casos y de los controles se utilizó un Kit de EnzymeLinked ImmunoSorbent Assay (ELISA) (Digestive Bio $\mathrm{K} 071{ }^{\circledR}$, Bio-X Diagnostics, Bélgica), que consiste en el uso de anticuerpos que permiten la captura específica del correspondiente antígeno, la sensibilidad y especificidad de acuerdo al laboratorio se encuentran entre $77 \%$ - $97 \%$ y entre 90 y $100 \%$, respectivamente. Para el procesamiento se siguieron las recomendaciones del fabricante. La lectura de la densidad óptica se realizó usando un lector de ELISA (BioStackReady ${ }^{\circledR}$ Lector BioTek ${ }^{\circledR}$ Power Wave XS) a $450 \mathrm{~nm}$ de longitud de onda. Los resultados se obtuvieron realizando una resta del valor de la muestra con el respectivo valor del control negativo y las muestras se consideran positivas cuando el valor fue superior a 0.150.

El diagnóstico de Salmonella se realizó mediante coprocultivo y caracterización usando un Kit de ELISA en "sandwich" que utiliza anticuerpos de captura específicos (Tecra ${ }^{\circledR}$ Salmonella Visual Immunoassay, Australia), una prueba utilizada inicialmente para detección de Salmonella en alimentos que mostró también buenos resultados en el diagnóstico del patógeno a partir de muestras de materia fecal en pequeños rumiantes (7). Se realizaron exámenes coprológicos usando la técnica de Ritchie o formol-éter que consiste en la sedimentación con una mezcla de solución salina formolada y éter, para el diagnóstico de Giardia sp (8) y la tinción de Ziehl Neelsen se utilizó para la confirmación de Criptosporidium sp. (8).

Análisis estadístico. Se utilizó estadística descriptiva de la zona de trabajo y de las variables relacionadas con los diferentes sistemas de manejo existentes en las explotaciones a nivel de finca, de ganado adulto y de los terneros.

Para llevar a cabo el análisis inferencial se utilizó como medida de asociación la prueba de $\chi^{2}$ (con un nivel de significancia de $\leq 0.05$ ). Posteriormente, se aplicaron modelos de regresión logística entre las variables seleccionadas buscando entre estos un modelo de buen ajuste por medio de la prueba de Hosmer \& Lemeshow, de este modelo se interpretaron los coeficientes odds ratio (9). Los datos fueron analizados con el programa de análisis estadístico SAS (SAS Institute Inc. 2003, SAS Guide for personal computers, Version 9.0 SAS Institute Inc., Cary, NC).

\section{RESULTADOS}

Se realizó el seguimiento de 620 animales, nacidos en 21 fincas ubicadas en 15 municipios, de los cuales el $10.3 \%(n=64)$ presentaron signos compatibles con DNB; se tomaron y evaluaron muestras de 68 animales controles pareados conjuntamente con las de los casos.

En las fincas enroladas en el presente estudio la raza predominante fue la Holstein, aunque también había animales de raza: Normando, Jersey, Pardo Suizo, Ayrshire, Angus y cruces entre estas.

La edad de presentación de diarrea fue la siguiente: en la primera semana de vida 19 casos $(29.7 \%)$, en la segunda $26(40.6 \%)$, en la tercera $10(15.6 \%)$, en la cuarta $3(4.7 \%)$ y en la quinta 6 $(9.47 \%)$. El porcentaje de distribución se presenta en la figura 1.

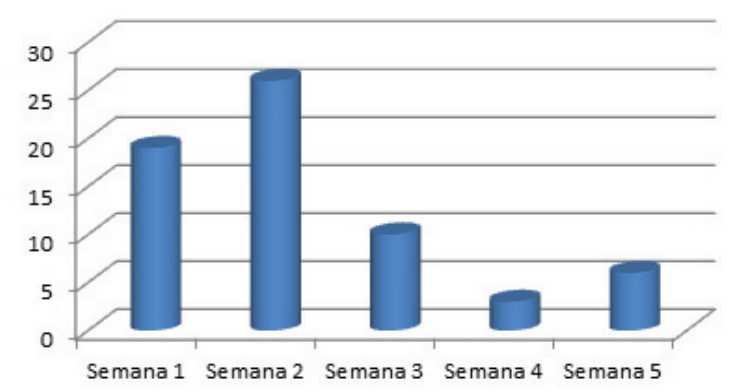

Figura 1. Edad de presentación de diarrea 
Se encontró en la prueba de ELISA de la totalidad de las muestras (casos y controles) que, 51 (38.3\%), $26(19,7 \%), 10(7.5 \%)$ y $1(0.75 \%)$ fueron positivas a Cryptosporidium sp, rotavirus, E coli $\mathrm{F} 5$ y coronavirus, respectivamente. La distribución de los resultados entre casos y controles se muestra en la Tabla 2.

Tabla 2. Distribución de muestras positivas a cada agente infeccioso en la prueba de ELISA.

\begin{tabular}{ccccc}
\hline & Rotavirus & Coronavirus & E coli F5 & Cryptosporidium \\
\hline Caso & 18 & 1 & 6 & 30 \\
Control & 8 & 0 & 4 & 21 \\
\hline Total & 26 & 1 & 10 & 51 \\
\hline
\end{tabular}

De los agentes estudiados, el único que se encontró en mayor cantidad de controles que en casos (6/3) fue Giardia sp. Con la prueba de Ziehl Neelsen se detectaron 19 casos y 6 controles positivos a Cryptosporidium sp (Figura 2).

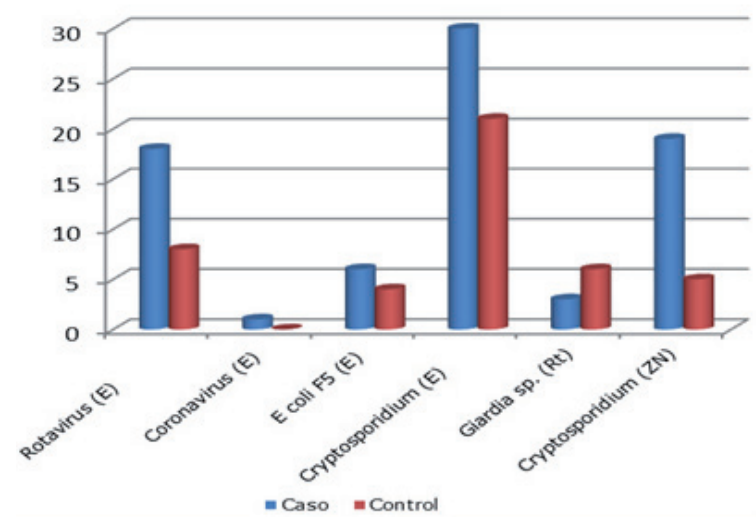

Figura 2. Distribución de muestras positivas a cada agente infeccioso en la prueba de ELISA (E), prueba de Ritchie (Rt) y tinción de Ziehl Neelsen (ZN).

Seis de los casos y dos de los controles presentaron infección combinada con Cryptosporidium sp., y rotavirus; 4 animales, 3 casos y 1 control fueron positivos para Cryptosporidium sp., y E. coli F5; sólo uno de los casos fue positivo para rotavirus y E. coli F5. Giardia sp., fue el agente encontrado con mayor frecuencia en los animales control $(n=6)$ comparado con los enfermos $(n=3)$ y sólo se encontró un caso positivo a coronavirus (Tabla 3). No se encontraron animales positivos a Salmonella sp.

De acuerdo con el análisis del modelo y de las pruebas de $\chi^{2}$ individuales, se evidencia que los dos agentes etiológicos que están asociados con la diarrea en los terneros son Cryptosporidium sp., $(p=0.001999)$ y Rotavirus $(p=0.031484)$ (Tabla 4).
Tabla 3. Infecciones combinadas e individuales de casos y controles.

\begin{tabular}{|c|c|c|c|c|}
\hline $\begin{array}{c}\text { AGENTES } \\
\text { INFECCIOSOS }\end{array}$ & CASO & $\begin{array}{c}\% \\
(n=132)\end{array}$ & CONTROL & $\begin{array}{c}\% \\
(n=132)\end{array}$ \\
\hline $\begin{array}{l}\text { Cryptosporidium sp. } \\
\text { (ELISA) }\end{array}$ & 30 & 22.73 & 21 & 15.91 \\
\hline ROTAVIRUS & 18 & 13.64 & 8 & 6.06 \\
\hline $\begin{array}{l}\text { Cryptosporidium sp. } \\
\text { (ELISA) y ROTAVIRUS }\end{array}$ & 6 & 4.55 & 2 & 1.52 \\
\hline $\begin{array}{l}\text { Cryptosporidium sp. y } \\
\text { E. Coli F5 }\end{array}$ & 3 & 2.27 & 1 & 0.76 \\
\hline ROTAVIRUS + E. Coli F5 & 1 & 0.76 & 0 & 0.00 \\
\hline E. Coli $\mathrm{F} 5$ & 6 & 4.55 & 4 & 3.03 \\
\hline CORONAVIRUS & 1 & 0.76 & 0 & 0.00 \\
\hline Giardia sp. & 3 & 2.27 & 6 & 4.55 \\
\hline No detección de agentes & 16 & 12.12 & 36 & 27.27 \\
\hline
\end{tabular}

Tabla 4. Resultados prueba $\chi^{2}$ para los agentes infecciosos.

\begin{tabular}{ll}
\hline \multicolumn{1}{c}{ Variable } & p valor \\
\hline Cryptosporidium (ZN) & 0.00199 \\
Giardia sp & 0.48825 \\
ROTAVIRUS & 0.03148 \\
CORONAVIRUS & 0.46826 \\
E. coli F5 & 0.52573 \\
Cryptosporidium (ELISA) & 0.07096 \\
\hline
\end{tabular}

Los resultados de la regresión logística muestran que los animales positivos para Rotavirus por la prueba de ELISA y para Cryptosporidium sp., por la técnica de Ziel Nielseen tuvieron respectivamente 2.6 y 7.0 veces mayor probabilidad que los demás animales de presentar DNB. En el modelo estadístico la interacción entre los agentes que resultaron factor de riesgo para la presentación de diarrea, no incremento la probabilidad de enfermar de los animales (Tabla 5).

Tabla 5. Agentes asociados con la presentación de DNB en fincas de la Sabana de Bogotá.

\begin{tabular}{lccc}
\hline \multicolumn{1}{c}{ Parámetro } & $\begin{array}{c}\text { Error } \\
\text { Standard }\end{array}$ & Pr $>\chi^{\mathbf{2}}$ & Odds \\
\hline Cryptosporidium (ZN) & 0.755 & 0.010 & 7.00 \\
ROTAVIRUS & 0.526 & 0.069 & 2.60 \\
Cryptosporidium (ZN)*Rotavirus & 11.755 & 0.042 & 0.09 \\
\hline
\end{tabular}

\section{DISCUSIÓN}

En el estudio se encontró que los agentes identificados con mayor frecuencia usando la prueba de ELISA, fueron Cryptosporidium sp., $(38.3 \%)$ y rotavirus $(19.7 \%)$, estos resultados son similares a los reportados en otros trabajos en los que se detecta la presencia de ooquistes de Cryptosporium sp., entre el $50 \%$ y $83 \%$ y la presencia de rotavirus en el $14.3 \%$, en terneros de 4 a 10 días de edad (10), otros estudios reportaron prevalencias entre $27.8 \%$ - 55\% y 17.7 - 58.7\% para Cryptosporidium sp., y rotavirus respectivamente (11-13). 
Varios autores han reportado la presencia de ooquistes de Cryptosporidium sp., en las heces de terneros, los mismos encontraron que los animales que excretan el protozoario tienen mayor probabilidad de presentar diarrea (1415); sin embargo, se debe tener en cuenta que el agente es detectado en un alto número de animales sanos y su presencia no siempre es causal de la enfermedad.

De acuerdo al modelo estadístico se encontró mayor asociación para la tinción de Ziehl Neelsen $(p<0.01)$ con el diagnóstico de terneros enfermos infectados con Cryptosporidium sp., comparado con el test de ELISA $(p<0.1)$. Sin embargo, este último detectó un mayor número de animales positivos para el protozoario; este hallazgo puede estar relacionado con la mayor capacidad del test de ELISA para detectar animales infectados con niveles más bajos de ooquistes (16), que no presentan cuadro clínico y mayor capacidad para la tinción de Ziehl Neelsen en la detección de animales enfermos que presentan mayor nivel de infección. Esto posiblemente se debe a la necesidad de que ocurra un grado de infestación parasitaria que pueda causar diarrea clínica y la prueba de la tinción de Ziehl Neelsen sólo los detecta cuando alcanzan este grado de infestación.

Estos resultados concuerdan con un estudio que reportó que terneros infectados con más de 2.2 $\times 10^{5}$ ooquistes de Cryptosporidium parvum/ gramo de materia fecal usando microscopia, tenían un mayor riesgo de presentar diarrea que los animales que excretaban niveles más bajos, confirmando que el número de ooquistes en las heces es un indicador de la intensidad de la infección (17).

La presencia de Rotavirus (por técnica de ELISA) y de Cryptosporidium sp., (por tinción de Ziehl Neelsen), son factores que incrementan el riesgo de desarrollo de la enfermedad; sin embargo la interacción de los agentes no incrementó la probabilidad de que los animales presentaran la enfermedad, posiblemente porque el efecto es ejercido individualmente o por el número escaso de infecciones combinadas presentes en el estudio.

A pesar de encontrar $E$. coli $\mathrm{F} 5$ en más casos que controles (6/4), no se encontró como un agente infeccioso asociado a la enfermedad. Los resultados se relacionan con otros estudios que reportan baja prevalencia del agente, esto puede deberse a que su presentación se limita a los primeros días de vida del ternero o a que se trata de un patógeno de baja frecuencia (11-
12), por tanto se debería revisar su impacto, teniendo en cuenta que en el presente estudio se diagnosticó en 7 animales mayores a una semana y sólo en 3 menores de 7 días de edad.

El análisis realizado para coronavirus no es concluyente ya que sólo se presentó una muestra positiva, que indica una baja frecuencia del agente en los animales de la zona de estudio. Sin embargo, resultados similares con relación a la prevalencia del coronavirus han sido reportados en estudios que observaron bajas prevalencias del agente (11-12-16).

La ausencia de resultados positivos para Salmonella sp., puede deberse a una muy baja incidencia, a la excreción intermitente de la bacteria en las heces, a baja sensibilidad de la prueba utilizada o a los métodos de conservación y procesamiento de la muestra en el laboratorio (18-19).

Aunque algunos reportes indican que hay evidencia clínica de la infección por Giardia sp., y su asociación con diarrea en terneros (20), los resultados encontrados no muestran dicha asociación; por el contrario se detectó el parásito en mayor cuantía en animales sanos que en los enfermos. La alta prevalencia reportada por otros estudios indica la presencia del agente en animales sanos (21-23) por tanto se debe investigar su importancia como enteropatógeno.

En el $25 \%(n=16)$ de los casos no se detectó alguno de los 5 agentes, resultados similares fueron reportados en España, donde tampoco encontraron agentes en el $19.7 \%$ de los animales. La explicación propuesta para ello es la presencia de causas no infecciosas de diarrea (causas nutricionales y otros factores de manejo), a fallas en el método detección en algunos animales positivos (en casos de excreción baja o intermitente del agente infeccioso) o a la presencia de agentes etiológicos menos comunes no investigados en el estudio (16). Sin embargo, otros estudios más recientes reportaron ausencia de animales positivos en un rango del $8.8 \%$ a $43.9 \%$ (1112 ). Por lo que se sugiere que en los futuros estudios sobre agentes causales de DNB se debe ampliar el espectro de agentes estudiados tanto infecciosos como nutricionales, entre otros.

Se puede concluir que la prevalencia de diarrea neonatal bovina en las fincas evaluadas fue del $10.3 \%$ y que se presentó con mayor frecuencia en terneros de dos semanas de edad. 
En el país no se han realizado estudios que determinen los agentes involucrados con la enfermedad, por tanto los resultados presentados son los primeros que muestran la importancia del Cryptosporidium sp., y del rotavirus en la DNB de las explotaciones ganaderas de Colombia.

\section{Agradecimientos}

A Intervet Schering-Plough Animal Health por el apoyo financiero para el desarrollo del proyecto. A los Laboratorios: Clínico, de Parasitología, Microbiología y de Virología de la Facultad de Medicina Veterinaria y de Zootecnia de la Universidad Nacional por su colaboración durante la investigación.

\section{REFERENCIAS}

1. Navarre C. Differentiation of gastrointestinal diseases of calves. Vet Clin Food Anim 2000; 6:37-57.

2. García A, Ruiz J, Orden J, Cid D, Sanz $\mathrm{R}$, Gómez-Bautista $M$ et al. Rotavirus and concurrent infections with other enteropathogens in neonatal diarrheic dairy calves in Spain. Comp Immunol Microbiol Infect Dis 2000; 23:175-183.

3. Scott $P$, Hall $G$, Jones $P$, Morgan J. Calf Diarrhoea. In Bovine Medicine. Diseases and Husbrandy of Cattle/edited by ANDREWS. Second Edition. Blackwell Science Ltd. Oxford 2004.

4. Radostits O, Gay C, Hinchcliff K, Constable P. Acute undifferentiated diarrhea of newborn farm animal. In Veterinary Medicine 10Th Ed.Saunders Elsevier 2007.

5. Escobar A, Bonilla R, Oliver O. Factores de manejo que afectan la morbilidad y mortalidad en terneros durante sus primeros tres meses de vida en el Municipio de San Pedro de los Milagros, departamento de Antioquia. Tesis de Grado. Facultad de Medicina Veterinaria y de Zootecnia. Universidad Nacional de Colombia. 1997.

6. Mejia G, Oliver O. Risks factors for morbidity and mortality in calves during the firsts four months of life in selected dairy herds in the high altitude tropic of Colombia XXIII World Buiatric Congress, Quebec City 12-16 July 2004.

7. Pao S, Patel D, Kalantari A, Tritschler J, Wildeus S, Sayre B. Detection of Salmonella strains and Escherichia coli 0157:H7 in feces of small ruminants and their isolation with various media. Appl Envir Microbiol 2005; 71:2158-2161.
8. Basso W, Venturini L, Risso M. Comparación de técnicas parasitológicas para el examen de heces de perro. Parasitología al día. $1998 ; 22: 1-2$.

9. Hosmer D, Lemeshow S. Applied logistic regression (Wiley series in probability and statistics), Canada: John Wiley \& Sons Inc; 2000.

10. Naciri M, Lefay $M$, Mancassola R, Poirier P, Chermette R. Role of Cryptosporidium parvum as a pathogen in neonatal diarrhoea complex in suckling and dairy calves in France. Vet Parasitol 1999; 85:245-257.

11. Lanz F, Kaufmann T, Sager S, Zanoni A, Schelling $E$, Meylan M. Prevalence of four enteropathogens in the faeces of young diarrhoeic dairy calves in Switzerland. The Vet Rec 2008; 163:362-366.

12. Bartels $C$, Holzhauer M, Jorritsma R, Swart W, Lam T. Prevalence, prediction and risk factors of enteropathogens in normal and non-normal faeces of young Dutch dairy calves. Pre Vet Med 2010; 93:162-169.

13. Torsein $M$, Lindberg $A$, Hallen $C$, Persson K, Törnquist M, Svensson C. Risk factors for calf mortality in large Swedish dairy herds. PREVET (2011), doi:10.1016/j. prevetmed.2010.12.001

14. Bagicha B, Sharma R, Kumar $H$, Rabinder $B$, Aulakh S, Singh J et al. Prevalence of Cryptosporidium parvum infection in Punjab (India) and its association with diarrhea in neonatal dairy calves. Vet Parasitol 2006; 140:162-165.

15. McAllister M. Protozoosis of the calf: Giardia, Cryptosporidium, Eimeria, Sarcocystis, Neospora. World Buiatrics Congress. Nice, France. 2006. 
16. De la Fuente $R$, Luzon M, Ruiz A, García A, Cid D, Orden J et al. Cryptosporidium and concurrent infections with other major enteropathogens in 1 to 30 day-old diarrheic dairy calves in central Spain. Vet Parasitol $1998 ; 14: 179-185$

17. Trotz L, Martin S, Leslie K, Duffield T, Nydam D, Peregrine A. Calf level risk factors for neonatal diarrhea and shedding of Cryptosporidium parvum in Ontario dairy calves. Pre Vet Med 2007; 82:12-28.

18. Warnick L, Kaneene J, Ruegg P, Wells S, Fossler C, Halbert $L$ et al. Evaluation of herd sampling for Salmonella isolation on midwest and northeast US dairy farms. Pre Vet Med 2003; 60:195-206.

19. Nielsen L, Schukken Y, Grohn Y, Ersboll A. Salmonella Dublin infection in dairy cattle: risk factors for becoming a carrier. Pre Vet Med 2004; 65:47-62
20. Geurden T, Claerebout E, Dursin L, Deflandre A, Bernay F, Kaltsatos $V$ et al. The efficacy of an oral treatment with paromomycin against an experimental infection with Giardia in calves. Vet Parasitol 2006; 135:241-247.

21. Trout J, Santín M, Greiner E, Fayer R. Prevalence of Giardia duodenalis genotypes in pre-weaned dairy calves. Vet Parasitol $2004 ; 124: 179-186$

22. Appelbee A, Frederick L, Heitman T, Olson $M$. Prevalence and genotyping of Giardia duodenalis from beef calves in Alberta, Canada. Vet Parasitol 2003; 112:289-294.

23. Hamnes I, Gjerde B, Robertson L. Prevalence of Giardia and Cryptosporidium in dairy calves in three areas of Norway. Vet Parasitol 2006; 140:204-216. 